\title{
Prospective Analysis of Patients with Axillary, Palmar and Axillary- Palmar Hyperhidrosis who Underwent Bilateral R4-R5 Video-Assisted Thoracoscopic Sympathicotomy
}

Jesús Alejandro Segura Martínez ${ }^{1}$, Agnaldo José Lopes ${ }^{2 *}$, Cláudio Higa1, Rodolfo Acatauassú Nunes ${ }^{1,2}$, Adriano Arnóbio José da Silva e Silva² and Eduardo Haruo Saito ${ }^{1,2}$

${ }^{1}$ Division of Thoracic Surgery, Pedro Ernesto University Hospital, State University of Rio de Janeiro, Rio de Janeiro, Brazil

${ }^{2}$ Postgraduate Programme in Medical Sciences, State University of Rio de Janeiro, Rio de Janeiro, Brazil

\begin{abstract}
Background: Video-assisted thoracoscopic sympathicotomy is a safe, effective and minimally invasive procedure, and its emergence greatly changed hyperhidrosis treatment. However, the possibility of standardizing interruption levels on the sympathetic chain has not yet been evaluated. This study sought to evaluate the frequency of compensatory sweating and the recurrence and satisfaction rates after bilateral R4-R5 video-assisted thoracoscopic sympathicotomy in patients with primary focal hyperhidrosis with involvement of the axillary, palmar, or both regions.
\end{abstract}

Methods: From November 2010 to February 2013, an observational prospective cohort study was conducted with 42 patients who underwent bilateral video-assisted thoracoscopic sympathicotomy. During the procedure, electrocautery was performed along the sympathetic chain at the levels R4-R5, and the nerve of Kuntz and the path between levels R4-R5 were also sectioned.

Results: No cases in which the video-assisted thoracoscopy had to be converted to thoracotomy were observed. Compensatory sweating was observed in $47.6 \%$ of patients within the first six months after surgery, decreasing to $30.9 \%$ at the end of this period. Hyperhidrosis recurrence occurred in only two patients. In total, $24.1 \%$ of patients with associated plantar hyperhidrosis reported that the condition disappeared after surgery. The satisfaction rate was $89.7 \%$ in the immediate postoperative period.

Conclusions: The frequency of compensatory sweating, the recurrence rate of symptoms and the satisfaction rate after sectioning by cauterization with electrocautery at the R4-R5 levels were similar to those reported for other types of interruption and other levels studied. Moreover, the interruption level adopted also had beneficial effects on plantar hyperhidrosis.

Keywords: Hyperhidrosis; Surgery; Sympathectomy; Electrocautery

\section{Introduction}

Hyperhidrosis is defined as a pathological condition of excessive sweating in amounts greater than physiologically necessary for thermoregulation, and this condition can be classified as primary or secondary $[1,2]$ and as focal or generalized. Focal hyperhidrosis is idiopathic and occurs more frequently in the palmar, plantar, axillary, inframammary, inguinal, and craniofacial regions $[1,2]$. The incidence of primary focal hyperhidrosis varies in different geographical areas and populations $[3,4]$. Women are generally more involved than men by this condition, and higher prevalence rates occur in adolescents and young adults $[3,4]$. Although no studies have described the natural course of the disease relative to age, Haider et al. [1] reported that the intensity of symptoms decreases in patients over 50 years of age.

The pathophysiology of hyperhidrosis is not entirely known, but it is accepted that hyperactive sweat glands are a result of exaggerated stimulation of the sympathetic autonomic nervous system. The eccrine glands are responsible for focal hyperhidrosis and are affected by emotional and gustatory stimuli. Although eccrine glands play a primary role in hyperhidrosis, some researchers believe that both eccrine and apocrine glands may have equal participation in axillary hyperhidrosis $[5,6]$.

There are several ways to treat hyperhidrosis, including the use of topic antiperspirants, iontophoresis, anticholinergics, beta-blockers, benzodiazepines, and botulinum toxin. However, clinical treatment options are not very satisfactory and have high recurrence rates $[1,3]$.
Conversely, surgical treatment with sympathectomy (resection of part of the sympathetic chain) or sympathicotomy (sectioning of levels of the sympathetic chain) is currently considered the method of choice [2]. The main side effects of sympathectomy include reflex or compensatory sweating, bradycardia, Horner's syndrome, chylothorax, hemothorax, pneumothorax, intercostal neuralgia, and recurrence [4].

Clinical history and physical examination are important tools to indicate the most appropriate individualized therapy in patients with hyperhidrosis. There are a number of clinical treatments for hyperhidrosis, including topical therapy, iontophoresis, systemic therapy, and botulinum toxin. Aluminum chloride and aluminum chlorohydrate salts are the most common topical treatments $[7,8]$. In addition to its limited effectiveness, the common side effects of topical

*Corresponding author: Agnaldo José Lopes, Postgraduate Programme in Medical Sciences, State University of Rio de Janeiro, Av. Prof. Manuel de Abreu, 444 Vila Isabel, 20550-170, Rio de Janeiro, Brazil, Tel: +55-21-21-25762030; E-mail: agnaldolopes.uerj@gmail.com

Received January 27, 2015; Accepted February 06, 2015; Published February 12, 2015

Citation: Martínez JAS, Lopes AJ, Higa C, Nunes RA, Silva AAJS, et al. Prospective Analysis of Patients with Axillary, Palmar and Axillary-Palmar Hyperhidrosis who Underwent Bilateral R4-R5 Video-Assisted Thoracoscopic Sympathicotomy. Journal of Surgery [Jurnalul de chirurgie] 2015; 11(1): 319-322 DOI: 10.7438/15849341-11-1-3

Copyright: (C) 2015 Martínez JAS, et al. This is an open-access article distributed under the terms of the Creative Commons Attribution License, which permits unrestricted use, distribution, and reproduction in any medium, provided the original author and source are credited. 
treatments (including burning sensation and local irritation) may reduce the benefits of treatment. A further alternative is iontophoresis, which has been reported to be effective in individuals that have both palmar and plantar hyperhidrosis. Iontophoresis requires long term use to maintain efficacy, necessitating home treatment; furthermore, this procedure is contraindicated in pregnant women, patients with a pacemakers or large metal prostheses, and who suffers from epilepsy $[9,10]$. The use of oxybutynin, an anticholinergic oral medication, is limited by the frequent presence of side effects, such as dry mouth, headache, constipation, and urinary retention. This type of treatment would, however, be suitable in cases which do not respond to topical treatments, botulinum toxin, or iontophoresis [11,12]. Botulinum toxin is effective, safe and has good tolerability as an alternative to topical, systemic, and surgical treatments; however, because primary hyperhidrosis is a chronic condition, questions about longevity of treatment efficacy remain [7,9]. When clinical options do not offer satisfactory results, a variety of surgical procedures can be used, including localized and thoracic surgery. Located surgery can only be used to treat axillary hyperhidrosis [7]. Although effective in resolving symptoms, this procedure often requires the placement of drains and is associated with a number of complications and side effects, including infection, hematoma, necrosis, atrophic or hypertrophic scarring, alopecia, and hyperpigmentation $[7,13]$.

The emergence of video-assisted thoracoscopic sympathicotomy greatly changed hyperhidrosis treatment, and this procedure is safe, effective, and minimally invasive [14,15]. In this procedure, the sympathetic chain is interrupted by sectioning or ablation of the nerve using electrocautery, a harmonic scalpel, or surgical clips. Despite the increasingly frequent use of video-assisted thoracoscopic sympathicotomy, evaluation of its long-term impact has been limited. Aiming to standardize this procedure, we believe that interrupting the sympathetic chain at the R4-R5 level in patients with hyperhidrosis in different regions may provide similar results as other types of interruption and other levels studied thus far. Therefore, the present study sought to evaluate the frequency of compensatory sweating and the recurrence and satisfaction rates after bilateral R4-R5 videoassisted thoracoscopic sympathicotomy in patients with primary focal hyperhidrosis with involvement of the axillary, palmar, or both regions.

\section{Materials and Methods}

\section{Patient population}

From November 2010 to February 2013, an observational prospective cohort study was conducted with 42 patients with primary focal hyperhidrosis (axillary, palmar or axillary-palmar). These patients underwent bilateral video-assisted thoracoscopic sympathicotomy with sectioning by cauterization with electrocautery along the sympathetic chain at the R4-R5 levels.

The criteria used to diagnose primary focal hyperhidrosis included focal sudoresis with visible and excessive sweating for a period $\geq 6$ months with no known clinical or drug cause combined with at least one of the following [6]: bilateral symmetrical disease; frequency of at least one episode per week; interference with daily activities; onset of symptoms before 25 years of age; family history of hyperhidrosis; and cessation of sweating during sleep.

In the preoperative period, all patients underwent a rigorous evaluation of their clinical history, electrocardiogram, and chest radiograph. The following were considered as contraindications for the procedure: secondary hyperhidrosis, body mass index $\geq 28 \mathrm{~kg} /$ $\mathrm{m}^{2}$, heart rate $<60 \mathrm{bpm}$, and radiological sequelae of pulmonary disease with probable signs of pleuropulmonary adhesions. After the surgical procedure, patient follow-up was performed during the first 24 hours, on days 15 and 30 and in the sixth month of the postoperative period, when the onset of compensatory sweating and/or recurrence of symptoms were evaluated, in addition to patient satisfaction. Compensatory sweating was classified based on the classification proposed by Atkinson et al. [16] as follows: absent (patient does not develop any new sweating in other areas); mild (patient develops new mild sweating in other areas, but it does not bother the patient); moderate (patient develops new sweating in other areas, but the patient still desires treatment); and severe (patient develops new sweating so serious that the patient regrets having undergone the procedure).

According to the World Medical Association Declaration of Helsinki, the study protocol was approved by the Research Ethics Committee of the State University of Rio de Janeiro, and written informed consent was obtained from all participants.

\section{Surgical procedure}

The procedure was performed with patients under general anesthesia. In some patients, orotracheal intubation was performed using a Robertshaw double lumen endobronchial tube (Broncho-Cath Left, Mallinckrodt Medical, Ireland), and in other patients, a simple tube with low-volume ventilation or small periods of apnea combined with carbon dioxide insufflators (maximum pressure of 8 to $10 \mathrm{mmHg}$ at a flow rate of $8 \mathrm{~L} / \mathrm{min}$ ) were used. Patients were then positioned in a semi-sitting supine position with a 45 -degree slope and with the upper limbs extended at a 90-degree angle relative to the body. An inframammary incision of approximately $1.5 \mathrm{~cm}$ was made in the anterior axillary line at the sixth intercostal space, followed by blunt dissection with penetration of the pleural cavity, and a 10-mm trocar was placed to introduce a rigid 30-degree, 10-mm optic (Karl-Storz, Tuttlingen, Germany) coupled to a miniature video camera (DX CAM Karl-Storz, Tuttlingen, Germany) to inspect the cavity and identify the sympathetic chain. Another incision of approximately $5 \mathrm{~mm}$ was made in the axillary region at the second intercostal space for placing a 5-mm trocar to introduce a hook clamp (Abbott Spine, Bordeaux, France) in the thoracic cavity with the aid of the rigid 30-degree optic. When the thoracic sympathetic chain was identified, a segment was sectioned by cauterization with electrocautery at the R4-R5 levels, as well as the path between levels R4-R5 and the nerve of Kuntz, whenever visualized. After the end of the procedure, a Levine tube (14 French) was introduced and water-sealed, and the lungs were expanded with Valsalva maneuvers until the air leak stopped. Then, the tube was removed with the lung in forced inspiration. The subcutaneous plane was closed with 2-0 polyglactin sutures, and the skin was closed with 4-0 polyglactin sutures. Subsequently, the same procedure was performed on the contralateral side. After the end of the procedure, patients were extubated and then underwent chest radiography for investigation of possible pneumothorax.

\section{Statistical analysis}

Descriptive statistics were used to analyze the data. The results were expressed as the median (minimum and maximum values) or number (percentage). Data analysis was performed using SAS 6.11 software (SAS Institute, Inc., Cary, NC, USA).

\section{Results}

Of the 42 patients studied, $61.9 \%$ were female, and the median age was 23.9 years at the time of surgery (ranging from 14 to 53 years). Before surgery, axillary, palmar and axillary-palmar hyperhidrosis was diagnosed in 4,17 , and 21 patients, respectively. Twenty-nine patients (69\%) complained of associated plantar hyperhidrosis.

All patients showed satisfactory results in the immediate postoperative period and on the first postoperative day, with dry hands and axillae. No cases of death related to surgery or cases in which the video-assisted thoracoscopy had to be converted to thoracotomy were 
observed. The median time spent in surgery was 40 minutes (ranging from 34 to 46 minutes), and the median hospitalization time was 3 days (ranging from 2 to 5 days). In the immediate postoperative period, one patient developed pneumothorax, and another patient showed mammary hypoesthesia.

Compensatory sweating was observed in 20 (47.6\%) patients within the first six postoperative months. Of these, 13 patients considered the compensatory sweating as mild, 3 as moderate, and 4 as severe. The following regions had the highest occurrence of compensatory sweating: trunk (8), thighs (6) and generalized form with involvement of the trunk, thighs, and buttocks (2). The onset of compensatory sweating was observed most often between days 15 and 30 of the postoperative period in $12(60 \%)$ of the 20 patients.

Regarding the initial area affected by hyperhidrosis before surgery, we observed that compensatory sweating appeared in the axillary region of $2(50 \%)$ of the 4 patients with axillary hyperhidrosis. Compensatory sweating appeared in the palmar region of $7(41.2 \%)$ of the 17 patients with palmar hyperhidrosis and in the axillary-palmar region of 11 (52.4\%) of the 21 patients with axillary-palmar hyperhidrosis.

At the end of the sixth month after surgery, 7 patients reported that the compensatory sweating had disappeared, whereas 9 did not report any improvement, although the clinical manifestations no longer bothered these patients. However, 4 patients complained that the compensatory sweating was more intense and uncomfortable, with no improvement. Among the 29 patients who had preoperative plantar hyperhidrosis associated with hyperhidrosis in other areas, 7 (24.1\%) reported that the plantar hyperhidrosis disappeared after surgery.

Of the 42 patients who received surgery, only 2 experienced hyperhidrosis recurrence. These subjects were initially included among the 4 patients who developed generalized and severe compensatory sweating and whose condition had not improved. In these patients, the recurrence began between the first and the sixth month after surgery, with progressive worsening during this time interval.

The satisfaction rate with the procedure was $89.7 \%$ in the first month and $76.9 \%$ in the sixth month. Among all the patients who received surgery, $89.7 \%$ reported they would recommend the surgical procedure to other patients with the same disease.

\section{Discussion}

The present study evaluated surgical interruption of the sympathetic chain segment at the R4-R5 levels in patients with axillary, palmar, or axillary-palmar hyperhidrosis, and the results were similar to those reported for other types of interruption and for other levels studied. In addition, the interruption level adopted in the present study also showed beneficial effects on plantar hyperhidrosis. To the best of our knowledge, this study is the first to demonstrate the possibility of standardizing a level for sectioning the sympathetic chain for surgical treatment of hyperhidrosis in different regions.

A wide array of therapies is available for the treatment of hyperhidrosis. It has been recommended that mild axillary hyperhidrosis is initially treated topically with aluminum salts or oxybutynin systemic therapy $[9,17,18]$. Botulinum toxin should be the second-line therapy in cases that do not respond to topical and systemic therapies [17]. In severe cases of axillary hyperhidrosis, botulinum toxin and topical aluminum chloride are first-line therapies [17]. The local axillary surgery should be considered after failure of other treatment options and before undergoing the patient to endoscopic thoracic surgery. Liposuction-curettage successfully relieves hyperhidrosis and, by virtue of the suctioning mechanism, may be superior to curettage alone for lasting results $[9,19]$. Surgical procedures are effective, safe, and permanent options for treating hyperhidrosis. Sympathectomy or sympathicotomy is advocated for debilitating hyperhidrosis of the extremities not responding to less invasive modalities [20]. However, these alternatives are reserved for severe cases, since they can have permanent side effects such as compensatory sweating [20].

In the present study, a segment of the sympathetic chain was interrupted by cauterization with electrocautery, and the path between levels R4-R5 and the nerve of Kuntz were sectioned. The most important factor related to this procedure was complete separation of the nerve as well as the space between the severed ends to prevent nerve regeneration. However, the level used for sympathetic sectioning remains controversial. In the present study, we used the Lin-Telaranta classification [21] to define specific section levels, in which the R3 and/ or R4 sections were proposed for palmar hyperhidrosis, and the R4 or R4-R5 sections were proposed for patients with axillary symptoms [21]. We chose to interrupt levels R4-R5 in all patients with axillary, palmar, and axillary-palmar complaints.

Considering that the purpose of hyperhidrosis surgery is to improve the patient's quality of life, complications should be minimal or preferably eliminated. In this sample, no cases of death resulting from surgery or cases in which video-assisted thoracoscopy had to be converted to thoracotomy were observed. The postoperative complications included pneumothorax and mammary hypoesthesia, which occurred in only two patients. This further highlights the success of the selected sympathetic chain interruption levels, and our results indicate the possibility of using the R4-R5 levels as a standard in all patients with axillary, palmar, and axillary-palmar symptoms.

Compensatory sweating is the most common adverse effect of sympathectomy, and its occurrence may vary between 3 and $98 \%$ $[22,23]$, with an average frequency of $60 \%$ described in the literature [24]. In some cases, the manifestation of this condition is transient and resolves after 6 to 12 months, although chronic cases may reach $67 \%$ according to Gossot et al. [24]. In the present study, $30.9 \%$ of patients remained with this complication after six months of follow-up, whereas $16.7 \%$ of patients reported the disappearance of compensatory sweating at the end of the sixth-month follow-up period. Regarding severe compensatory sweating, the frequency reported in the literature varies but was shown to reach $36 \%$ of cases in one report [25]. However, according to Montessi et al. [26], the frequency of severe compensatory sweating may vary depending on the level sectioned, with rates of $32 \%$ after cauterization at the R2 level, 9\% after cauterization at the R3 level, and only $4 \%$ after cauterization at the R4 level. In the present study, only 4 of the 42 patients reported severe compensatory sweating.

The hyperhidrosis recurrence rate after video-assisted thoracoscopic surgery reported in the literature varies between $0 \%$ and $65 \%[27,28]$. In the present study, it was observed that only two patients experienced hyperhidrosis recurrence; one patient had an initial complaint of axillary hyperhidrosis, and the other complained of axillary-palmar hyperhidrosis. The recurrence rates reported in the literature for focal axillary hyperhidrosis vary between 15 and $65 \%[24,29]$, whereas the overall recurrence rate of palmar hyperhidrosis varies between zero and $16 \%$ [30]. However, it is important to investigate whether such case are related to the presence of secondary hyperhidrosis, so that the possible causes of recurrence can be identified. Interestingly, Lin et al. [31] found that the causes for recurrence were neural regeneration in $18.8 \%$ of cases, incomplete disruption of the R2 node in $12.5 \%$ of cases and integrity of the nerve of Kuntz in $18.8 \%$ of cases.

Previous studies including a series of patients treated with sympathectomy for primary hyperhidrosis showed a high degree of patient satisfaction. In our study, the satisfaction rate was $89.7 \%$ in the first month and $76.9 \%$ in the sixth month after surgery. We believe that this reduction in the satisfaction rate during the follow-up period may be attributed to the emergence of compensatory sweating and/or 
recurrence. In the literature, the best results were reported by Cohen et al. [32], with a satisfaction rate of $98.2 \%$. Surprisingly, in the present study, $24.1 \%$ of patients with complaints of plantar hyperhidrosis associated with hyperhidrosis in other areas reported that the plantar abnormality disappeared after surgery. This finding was also reported by Gossot et al. [24], who found that $15 \%$ of patients described an improvement in plantar-associated symptoms, and some patients were even considered cured after the procedure.

A critical analysis of the results and their limitations is important. The present study was conducted with a small number of patients and only investigated the interruption of the sympathetic chain at the R4-R5 levels. Furthermore, we evaluated only subjects with axillary, palmar, or palmar-axillary hyperhidrosis. Despite these limitations, our results suggest that it is possible to standardize the levels for sectioning the sympathetic chain for surgical treatment of hyperhidrosis in different regions. We believe that the present study represents an important contribution to the field because it may serve as a starting point for future clinical trials with greater numbers of patients and other sympathetic chain interruption levels.

In conclusion, the present study shows that bilateral videoassisted thoracoscopic sympathicotomy at the R4-R5 levels is a safe procedure that provides good results in the treatment of primary focal hyperhidrosis with axillary, palmar and axillary-palmar involvement. The frequency of compensatory sweating, the recurrence rate of symptoms and the satisfaction rate found for sectioning by cauterization with electrocautery at the R4-R5 levels were similar to those previously reported in the literature for other types of interruption and other levels studied. Moreover, the sympathetic chain interruption levels used in the present study also showed beneficial effects on plantar hyperhidrosis.

\section{Conflict of Interest}

The authors have no conflict of interest to report.

\section{Acknowledgements}

The authors wish to thank the Rio de Janeiro State Research Supporting Foundation (FAPERJ), Rio de Janeiro, Brazil.

\section{References}

1. Haider A, Solish N (2005) Focal hyperhidrosis: diagnosis and management. CMAJ 172: 69-75.

2. Shields TW, Locicero J (2009) General thoracic surgery. (7th ed), Lippincott Williams \& Wilkins, Philadelphia.

3. Walling HW, Swick BL (2011) Treatment options for hyperhidrosis. Am J Clin Dermatol 12: 285-295.

4. Cerfolio RJ, De Campos JR, Bryant AS, Connery CP, Miller DL, et al. (2011) The Society of Thoracic Surgeons expert consensus for the surgical treatment of hyperhidrosis. Ann Thorac Surg 91: 1642-1648.

5. Lonsdale-Eccles A, Leonard N, Lawrence C (2003) Axillary hyperhidrosis: eccrine or apocrine? Clin Exp Dermatol 28: 2-7.

6. Hornberger J, Grimes K, Naumann M, Glaser DA, Lowe NJ, et al. (2004) Recognition, diagnosis, and treatment of primary focal hyperhidrosis. J Am Acad Dermatol 51: 274-286.

7. Gontijo GT, Gualberto GV, Madureira NAB (2011) Axillary hyperhidrosis treatment update. Surg Cosmet Dermatol 3: 47-51.

8. Reisfeld R, Berliner KI (2008) Evidence-based review of the nonsurgical management of hyperhidrosis. Thorac Surg Clin 18: 157-166.

9. Stashak AB, Brewer JD2 (2014) Management of hyperhidrosis. Clin Cosmet Investig Dermatol 7: 285-299.

10. Cohen JL, Cohen G, Solish N, Murray CA (2007) Diagnosis, impact, and management of focal hyperhidrosis: treatment review including botulinum toxin therapy. Facial Plast Surg Clin North Am 15: 17-30.

11. Wolosker N, Teivelis MP, Krutman M, de Paula RP, Kauffman P, et al (2014) Long-term results of the use of oxybutynin for the treatment of axillary hyperhidrosis. Ann Vasc Surg 28: 1106-1112.
12. Wolosker N, Krutman M, Teivelis MP, Paula RP, Kauffman P, et al. (2014) Analysis of oxybutynin treatment for hyperhidrosis in patients aged over 40 years. Einstein (Sao Paulo) 12: 42-47.

13. Schlereth T, Dieterich M, Birklein F (2009) Hyperhidrosis--causes and treatment of enhanced sweating. Dtsch Arztebl Int 106: 32-37.

14. Drott C, Göthberg G, Claes G (1995) Endoscopic transthoracic sympathectomy: an efficient and safe method for the treatment of hyperhidrosis. J Am Acad Dermatol 33: 78-81.

15. Lopes AC (2003) Topics in clinical medicine. Medsi, Rio de Janeiro.

16. Atkinson JL, Fode-Thomas NC, Fealey RD, Eisenach JH, Goerss SJ (2011) Endoscopic transthoracic limited sympathotomy for palmar-plantar hyperhidrosis: outcomes and complications during a 10-year period. Mayo Clin Proc 86: 721-729.

17. Solish N, Bertucci V, Dansereau A, Hong HC, Linde C, et al. (2007) A comprehensive approach to the recognition, diagnosis, and severitybased treatment of focal hyperhidrosis: recommendations of the Canadian Hyperhidrosis Advisory Committee. Dermatol Surg 33: 908-923.

18. Wolosker N, de Campos JR, Kauffman P, Puech-Leão P (2012) A randomized placebo-controlled trial of oxybutynin for the initial treatment of palmar and axillary hyperhidrosis. J Vasc Surg 55: 1696-1700.

19. Tronstad C, Helsing P, Tønseth KA, Grimnes S, Krogstad AL (2014) Tumescent suction curettage vs. curettage only for treatment of axillary hyperhidrosis evaluated by subjective and new objective methods. Acta Derm Venereol 94: $215-220$.

20. Lee KY, Levell NJ (2014) Turning the tide: a history and review of hyperhidrosis treatment. JRSM Open 5: 2042533313505511.

21. Lin CC, Telaranta T (2001) Lin-Telaranta classification: the importance of different procedures for different indications in sympathetic surgery. Ann Chir Gynaecol 90: 161-166.

22. Lyra Rde M, Campos JR, Kang DW, Loureiro Mde P, Furian MB, et al. (2008) Guidelines for the prevention, diagnosis and treatment of compensatory hyperhidrosis. J Bras Pneumol 34: 967-977.

23. Sugimura H, Spratt EH, Compeau CG, Kattail D, Shargall Y (2009) Thoracoscopic sympathetic clipping for hyperhidrosis: long-term results and reversibility. J Thorac Cardiovasc Surg 137: 1370-1376.

24. Gossot D, Galetta D, Pascal A, Debrosse D, Caliandro R, et al. (2003) Long-term results of endoscopic thoracic sympathectomy for upper limb hyperhidrosis. Ann Thorac Surg 75: 1075-1079.

25. de Campos JR, Kauffman P, Werebe Ede C, Andrade Filho LO, Kusniek S, et al. (2003) Quality of life, before and after thoracic sympathectomy: report on 378 operated patients. Ann Thorac Surg 76: 886-891.

26. Montessi J, Almeida EP, Vieira JP, Abreu Mda M, Souza RL, et al. (2007) Videoassisted thoracic sympathectomy in the treatment of primary hyperhidrosis: a retrospective study of 521 cases comparing different levels of ablation. J Bras Pneumol 33: 248-254.

27. Yazbek G, Wolosker N, Kauffman $P$, Campos JR, Puech-Leão $P$, et al. (2009) Twenty months of evolution following sympathectomy on patients with palmar hyperhidrosis: sympathectomy at the T3 level is better than at the T2 level Clinics 64: 743-749.

28. Yazbek G, Wolosker N, de Campos JR, Kauffman P, Ishy A, et al. (2005) Palmar hyperhidrosis--which is the best level of denervation using videoassisted thoracoscopic sympathectomy: T2 or T3 ganglion? J Vasc Surg 42 281-285.

29. Claes G (2003) Indications for endoscopic thoracic sympathectomy. Clin Auton Res 13 Suppl 1: I16-19.

30. Yano M, Fujii Y (2006) Endoscopic thoracic sympathectomy for palmar hyperhidrosis. Ann Thorac Cardiovasc Surg 12: 81-82.

31. Lin TS (2001) Video-assisted thoracoscopic "resympathicotomy" for palmar hyperhidrosis: analysis of 42 cases. Ann Thorac Surg 72: 895-898.

32. Cohen Z, Levi I, Pinsk I, Mares AJ (1998) Thoracoscopic upper thoracic sympathectomy for primary palmar hyperhidrosis-the combined paediatric, adolescent and adult experience. Eur J Surg 580:5-8. 